\title{
To predict human choice, consider the context
}

\author{
Ori Plonsky* and Ido Erev
}

Technion

*Correspondence: plonsky@technion.ac.il

\begin{abstract}
.
Choice prediction competitions suggest that popular models of choice, including prospect theory, have low predictive accuracy. Peterson et al. show the key problem lies in assuming each alternative is evaluated in isolation, independently of the context. This observation demonstrates how a focus on predictions can promote understanding of cognitive processes.
\end{abstract}

Keywords:

Machine learning, Reliance on small samples, BEAST, decisions under risk, decisions under uncertainty. 
Studies of risky choice highlight a large difference between the most popular descriptive models, including prospect theory [1], and models that perform best when evaluated based on out-of-sample predictive accuracy in choice prediction competitions (open tournaments in which researchers submit quantitative models aimed to predict unseen data of human choice) $[2,3]$. The popular models assume that people evaluate each risky alternative by weighting the subjective values of its possible outcomes by subjective functions of their probabilities. The best models in the competitions assume that the main driver of choice is a tendency to rely on small samples of feasible scenarios. The key difference between these assumptions involves the impact of the context. Prospect theory and similar models imply that each alternative is evaluated in isolation, independently of the other available alternatives. In contrast, reliance on small samples of scenarios implies a context effect: large sensitivity to the proportion of scenarios in which each alternative provides the best payoff (Box 1).

In a clever study, Peterson et al. [4] (who also won a recent competition) build on machine learning methods to establish the importance of context. They collected an extremely large dataset (9831 experimental conditions) and used it to train deep neural networks of various structures, gradually allowing them more flexibility. For example, one network structure could only learn a subjective function of each alternative's outcomes (as in expected utility theory, EUT) while a less restrictive structure could also learn a subjective weighting of probabilities (as in prospect theory). The least restrictive structure allows the networks to learn functions by the context of the problem. Because deep neural networks can in principle learn any function of the inputs, this process effectively produced the optimal models among all models that adhere to each level of restrictions imposed. These models were then compared based on their out-ofsample prediction accuracy. Their results show that assuming people evaluate the alternatives in isolation is far too restrictive. To provide good predictions of behavior, models must account for the context in which a choice is made.

Peterson et al. continue to suggest one possible way by which context may impact choice. Their Mixture of Theories (MOT) model assumes context determines a utility function and a subjective weighting function, both of which are weighted mixtures of one version of expected utility and one version of prospect theory. These are then combined to assign subjective values to each alternative. Additionally, MOT assigns a fixed prediction when one alternative dominates the other. MOT gives excellent predictions when trained on relatively little data. Importantly 
however, Peterson et al. do not argue that MOT is necessarily the best abstraction. Indeed, there are simpler models that account for context and provide similarly good predictions. One example is a minor refinement of Best Estimate and Sampling Tools (BEAST), the model on which winners of both latest choice prediction competitions were based. Without additional training or modifications, BEAST (developed to predict choices of students in a slightly different experimental paradigm) outperforms all theoretical models considered by Peterson et al., but falls short of MOT, predicting far more "rational behavior" than the data (choices of MTurk participants) suggest. The modified version allows for more violations of dominance and for less sensitivity to the difference between expected values. The out-of-sample predictions' mean squared errors of both MOT and modified BEAST is just under 0.012, roughly half of those of other, more traditional, models. Furthermore, BEAST has additional advantages: It is easily interpretable (it rests on the reliance on small samples hypothesis explained in Box 1), and has predictions that go beyond the scope of these data (ambiguous problems, dynamics of choice with feedback).

Recent research highlighted how cognitive and behavioral research can benefit from common practices used in computational sciences [5,6]. However, adoption of these practices is slow, partly because researchers believe a focus on predictions rather than on interpretable causal explanations is unlikely to promote scientific understanding. Peterson et al.'s observations, and the competitions' results, suggest at least three ways by which prediction-oriented research can advance cognitive research and promote understanding.

The first involves the method used to reduce the risk of overfitting the data. Traditionally, model developers try to reduce this risk by focusing on refinements of existing models. For example, prospect theory explains violations of EUT by refining the abstraction of the assumed weighting functions, but without modifying the basic idea (isolated evaluation of each alternative with a subjective function of the input). To clarify the implication of this "gradual adjustment process", it is convenient to think about model development as a hike in a "land of assumptions" in an attempt to find a hill that provides good view of the "land of behaviors" [7]. When the hike starts at a low point on a hill of high elevation, the gradual adjustment process is effective. However, if the starting point is on a hill of low elevation, gradual adjustment is too restrictive. The evaluation of models based on out-of-sample predictive accuracy in large datasets can help find hills of higher elevation while guarding against overfitting. 
The second is the possibility to disentangle theoretical assumptions from their mathematical implementations. Machine learning systems are far better than humans at approximating a function given the inputs. Researchers can focus on constructing theoretically relevant inputs and outsource the job of finding the best function of those inputs to automated processes [8]. By finding the best possible implementation from a class of models in a datadriven manner, it then becomes easier to compare wide classes of models at once. Peterson et al. demonstrate how the class of isolated evaluation models can be compared with the larger class of models that are sensitive to context.

The third involves the idea that, in theory, unconstrained machine learning models trained on large datasets can identify what patterns in the data are predictable and approximate the true underlying function better than the raw data [9]. Hence, they can be used to guide researchers towards the assumptions that should be added to simple cognitive models to improve predictions, while preserving insights from classical research, as Peterson et al. demonstrate.

\section{Box 1: Example of the impact of context}

Consider a choice between daily locations for a food truck. Location A yields a gain of 8 in rainy days (50\% of the days), but 0 in other days. Location B yields a gain of only 6 in rainy days, and 0 in other days. Location $\mathrm{C}$ is a mirror image of $\mathrm{B}$, it pays 0 in rainy days, and 6 in other days. The truck driver chooses either between locations A or B (context AorB) or between locations A or $\mathrm{C}$ (context AorC). Hence, $\mathrm{A}$ is considered under one of two contexts. Because $\mathrm{B}$ and $\mathrm{C}$ are associated with the same payoff distributions, isolated evaluation of each option implies the same choice rates in the two contexts. In contrast, experimental studies [2] suggest more choice of A when the alternative is $\mathrm{B}$ than when it is $\mathrm{C}$. This pattern can be explained by assuming reliance on small samples of possible scenarios. While Option B in never better than A, Option C is better than $\mathrm{A}$ in half of the days, and when the sample is small $\mathrm{C}$ can appear more attractive than A. 


\section{References}

1 Kahneman, D. and Tversky, A. (1979) Prospect theory: An analysis of decision under risk. Econometrica 47, 263-292

2 Erev, I. et al. (2017) From anomalies to forecasts: Toward a descriptive model of decisions under risk, under ambiguity, and from experience. Psychol. Rev. 124, 369-409

3 Plonsky, O. et al. (2019) Predicting human decisions with behavioral theories and machine learning. arXiv Prepr. arXiv1904.06866

4 Peterson, J.C. et al. (2021) Using large-scale experiments and machine learning to discover theories of human decision-making. Science (80-. ). 372, 1209-1214

5 Hofman, J.M. et al. (2021) Integrating explanation and prediction in computational social science. Nature 595, 181-188

6 Yarkoni, T. and Westfall, J. (2017) Choosing prediction over explanation in psychology: Lessons from machine learning. Perspect. Psychol. Sci. 12, 1100-1122

7 Erev, I. (2020) Money makes the world go round, and basic research can help. Judgm. Decis. Mak. 15, 304-310

8 Plonsky, O. et al. (2017), Psychological forest: Predicting human behavior., in 31st AAAI Conference on Artificial Intelligence, AAAI 2017

9 Agrawal, M. et al. (2020) Scaling up psychology via scientific regret minimization. Proc. Natl. Acad. Sci. 117, 8825-8835 